OPEN ACCESS

Edited by:

Marina I. Arleevskaya,

Kazan State Medical

Academy, Russia

Reviewed by:

Mario M. D'Elios,

University of Florence, Italy

Francois-Pierre Martin,

Nestle Institute of Health Sciences,

Switzerland

Arne Yndestad,

Oslo University Hospital, Norway

*Correspondence:

Clara G. de los Reyes-Gavilán greyes_gavilan@ipla.csic.es

Specialty section:

This article was submitted to

Microbial Immunology,

a section of the journal

Frontiers in Immunology

Received: 07 April 2017

Accepted: 29 June 2017

Published: 24 July 2017

Citation:

Rodríguez-Carrio J, Salazar N

Margolles A, González S,

Gueimonde $M$, de los Reyes-

Gavilán CG and Suárez A (2017) Free

Fatty Acids Profiles Are Related to

Gut Microbiota Signatures and

Short-Chain Fatty Acids.

Front. Immunol. 8:823.

doi: 10.3389/fimmu.2017.00823

\section{Free Fatty Acids Profiles Are Related to Gut Microbiota Signatures and Short-Chain Fatty Acids}

\author{
Javier Rodríguez-Carrio', Nuria Salazar', Abelardo Margolles', Sonia González², \\ Miguel Gueimonde', Clara G. de los Reyes-Gavilán ${ }^{1 \star}$ and Ana Suárez ${ }^{3}$
}

'Department of Microbiology and Biochemistry of Dairy Products, Instituto de Productos Lácteos de Asturias (IPLA-CSIC), Villaviciosa, Asturias, Spain, ${ }^{2}$ Area of Physiology, Department of Functional Biology, University of Oviedo, Oviedo, Asturias, Spain, ${ }^{3}$ Area of Immunology, Department of Functional Biology, University of Oviedo, Oviedo, Asturias, Spain

A growing body of evidence highlights the relevance of free fatty acids (FFA) for human health, and their role in the cross talk between the metabolic status and immune system. Altered serum FFA profiles are related to several metabolic conditions, although the underlying mechanisms remain unclear. Recent studies have highlighted the link between gut microbiota and host metabolism. However, although most of the studies have focused on different clinical conditions, evidence on the role of these mediators in healthy populations is lacking. Therefore, we have addressed the analysis of the relationship among gut microbial populations, short-chain fatty acid (SCFA) production, FFA levels, and immune mediators (IFN $\gamma, \mathrm{IL}-6$, and MCP-1) in 101 human adults from the general Spanish population. Levels of selected microbial groups, representing the major phylogenetic types present in the human intestinal microbiota, were determined by quantitative PCR. Our results showed that the intestinal abundance of Akkermansia was the main predictor of total FFA serum levels, displaying a negative association with total FFA and the pro-inflammatory cytokine IL-6. Similarly, an altered FFA profile, identified by cluster analysis, was related to imbalanced levels of Akkermansia and Lactobacillus as well as increased fecal SCFA, enhanced IL-6 serum levels, and higher prevalence of subclinical metabolic alterations. Although no differences in nutritional intakes were observed, divergent patterns in the associations between nutrient intakes with intestinal microbial populations and SCFA were denoted. Overall, these findings provide new insights on the gut microbiota-host lipid metabolism axis and its potential relevance for human health, where FFA and SCFA seem to play an important role.

Keywords: free fatty acids, microbiota, Akkermansia, short-chain fatty acids, serum lipids, subclinical metabolic alterations

\section{INTRODUCTION}

Free fatty acids (FFA) are lipid species released from the adipose tissue and several cell types upon lipolysis. Apart from their classical roles in energy supply or as structural components, FFA are emerging as active players of a number of biological processes. FFA can affect gene expression of macrophages (1), adipocytes (2), or endothelial cells (3). In addition, FFA can modulate the production of chemokines and cytokines (3-5), the expression of genes coding for adhesion molecules $(6,7)$ and they give rise to pro-inflammatory and inflammation pro-resolving lipid-derived species (8). 
Therefore, a growing body of evidence emphasizes a role for FFA as common mediators between metabolic conditions and the immune system. FFA have been proposed as a mechanistic explanation for the relationship among obesity, inflammation, altered glucose homeostasis, and cardiovascular disease (9). Similarly, some FFA have been reported as regulators of systemic metabolism homeostasis in mice (10). Importantly, quantitative and qualitative differences among the effects of individual FFA have been revealed (11). Thus, not only increased levels but also an altered FFA pool composition may be associated with the risk of developing a range of disorders in which the immune system plays a role (12-17). However, the underlying causes of the altered FFA pool composition remain unknown.

Compelling evidence from recent years has shed some light on the effects that gut microbiota can exert on the host health. Alterations in the composition of the intestinal microbiota have been related with the development of metabolic disorders both in mice and in human studies (18), whereas some dietary factors are also known to modulate these microbial communities (19). Recent studies indicate that the gut microbiota is also involved in the host energy metabolism by regulating the absorption of nutrients, local production of hormones and immune mediators, fat storage, and gut permeability (20-22). However, to what extent host lipid metabolism is associated with the intestinal microbial populations, and whether the gut microbiota could be related to FFA levels, remains largely unknown. Short-chain fatty acids (SCFA) are produced in the gut by the metabolic activity of the intestinal microbiota as catabolic end-products from the fermentation of undigested dietary components, mainly complex carbohydrates. These compounds are pivotal in the interactions between the host and intestinal microbial populations (23), but their actual links with the metabolism of fatty acids in humans are controversial. In addition, since most studies have been performed with relatively low sample sizes and mainly with $a$ priori established conditions, evidence for their potential relevance in healthy adults is lacking in the literature. However, due to their pivotal role in shaping the host metabolism, it is tempting to speculate that the intestinal microbiota could be related to FFA levels in healthy populations.

Based on these lines of evidence, we hypothesize that alterations in the composition and metabolic activity of the gut microbiota may be associated with altered levels of FFA, which can be in turn be related with some mediators of inflammation. With the aim to gain insight into the joint impact of these players in human health, we have studied the relationships among selected microbial populations, fecal SCFA, serum FFA levels, and composition, as well as inflammatory mediators. In this way, the main objective of the present study was to evaluate whether specific gut microbial signatures can be related to altered levels of FFA species in adults from the general population.

\section{MATERIALS AND METHODS}

\section{Ethical Approval}

Ethical approval for this study was obtained from the Institutional Review Board (Comité de Ética de Investigación Clínica del Principado de Asturias) in compliance with the Declaration of Helsinki. All participants were informed and gave a signed informed consent prior their inclusion in the study.

\section{Subjects}

A group of 101 individuals was recruited from the general population in the Asturias region, northern Spain. Exclusion criteria were the diagnosis of chronic or immune-mediated diseases, recent infections, cancer, or altered metabolic conditions, as well as the current usage (within the previous 6 months) of immunomodulatory drugs, metabolic agents, probiotics, or antibiotics. Demographical parameters of the analyzed population are summarized in Table 1.

TABLE 1 | Description of the study population.

Study population $(n=101)$
Demographical parameters

Age, mean (range)

Gender (F/M)

$\mathrm{BMl}$

Biochemical parameters (mg/dl)

Glucose

Total cholesterol

HDL cholesterol

LDL cholesterol

Triglycerides

Microbial populations (log cells/g)

Akkermansia

Bacteroides group

Bifidobacterium

Clostridium cluster XIVa

Lactobacillus group

Faecalibacterium

Main short-chain fatty acid (SCFA) (mM)

Acetate

Propionate

Butyrate

Total SCFA

\section{Free fatty acids (FFA) $(\mu \mathrm{g} / \mathrm{ml})$}

Palmitic (16:0)

Stearic (18:0)

Palmitoleic (16:1w7)

Oleic (18:1w9)

Linoleic (18:2w6)

$\gamma$-Linoleic (18:3w6)

AA (20:4w6)

Linolenic (18:3w3)

Eicosapentaenoic (20:5w3)

Docosahexaenoic acid (22:6w3)

SFA

MUFA

W6-PUFA

w3-PUFA

Total FFA (mM)

Cytokine serum levels $(\mathrm{pg} / \mathrm{ml})$

IL-6

MCP-1

IFN $\gamma$

Variables are summarized as median (interquartile range), mean $\pm S D$, or $n(\%)$, as

appropriated, unless otherwise stated.
$49.41(19-72)$ $66 / 35$

$26.76 \pm 4.39$

$96.49 \pm 12.20$

$208.66 \pm 41.64$

$56.70 \pm 12.84$

$130.41 \pm 35.80$

$107.53 \pm 62.78$

$5.84 \pm 1.80$

$9.15 \pm 1.16$

$8.26 \pm 0.91$

$8.52+1.41$

$6.14 \pm 1.04$

$8.05 \pm 1.29$

$40.46 \pm 19.20$

$15.04 \pm 8.34$

$10.61 \pm 7.19$

$66.42 \pm 31.06$

$28.34(12.04)$

$28.35(9.98)$

$0.80(1.20)$

$27.38(20.12)$

$7.90(7.11)$

$0.08(0.08)$

$2.02(1.49)$

$0.18(0.16)$

$0.09(0.16)$

$1.31(1.59)$

$58.28(19.08)$

$28.15(20.48)$

$10.59(8.21)$

$1.67(1.74)$

$0.31(0.17)$

442.79 (949.99)

$267.30(127.83)$

$2.05(4.70)$ 
Subjects were asked to participate in this study and, upon acceptance, an overnight fast blood sample was drawn by venipuncture in tubes without anticoagulant. After blood clotting, serum was collected and stored at $-80^{\circ} \mathrm{C}$ until further analyses. In addition, basic serum biochemical analyses were performed by standardized procedures. Subjects were considered to exhibit a subclinical metabolic alteration if they meet any of the following criteria: fasting glucose levels higher than $100 \mathrm{mg} / \mathrm{dl}$ or that of triglycerides over $150 \mathrm{mg} / \mathrm{dl}$. These objectives cut offs were obtained from clinical national guidelines.

\section{Total FFA Assessment}

Total FFA serum levels were quantified by means of a colorimetric assay using a commercial kit (NEFA kit half-microtest, Roche Life Sciences, Penzberg, Germany) following the protocol from the manufacturer. Final absorbance was measured at $546 \mathrm{~nm}$, and detection limit was $0.02 \mathrm{mM}$.

\section{Individual FFA Quantification}

Free fatty acids were analyzed after a methyl-tert-butylether (MTBE)-based extraction protocol as previously described (24) with slight modifications. Briefly, $100 \mu \mathrm{l}$ serum samples were spiked with $5 \mu \mathrm{l}$ of internal standard (600 ppm heptadecanoic acid). Then, protein precipitation was performed by adding $200 \mu \mathrm{l}$ methanol chromasolv grade (Sigma Aldrich, MO, USA), and tubes were vortexed for $30 \mathrm{~s}$. Then, 1,200 $\mu \mathrm{l} \mathrm{MTBE}$ chromasolv grade (Sigma) was added, vortexed, and an incubation in an ultrasound water bath at $15^{\circ} \mathrm{C}$ was performed for $30 \mathrm{~min}$. Finally, organic phase was separated after the addition of $200 \mu \mathrm{l}$ milliQ water and a centrifugation step for $7 \mathrm{~min}$ at 5,000 rpm $\left(15^{\circ} \mathrm{C}\right)$. After collecting the upper layer, the extraction protocol was repeated once with $100 \mu \mathrm{l} \mathrm{MetOH,} 500 \mu \mathrm{l} \mathrm{MTBE}$, and $100 \mu \mathrm{l}$ milliQ $\mathrm{H}_{2} \mathrm{O}$.

Lipid extracts were dried in a miVac centrifugal evaporator (Genevac Ltd., UK) and redissolved in $100 \mu \mathrm{l}$ of water:acetonitrile 38:62.

For the determination of the fatty acids, a Dionex Ultimate 3000 HPLC system (Thermo Scientific, Bremen, Germany), consisting of a high pressure binary pump, an autosampler and a column oven, was used. The column was a Zorbax Eclipse Plus C18, $50 \mathrm{~mm} \times 2.1 \mathrm{~mm}, 1.8 \mu \mathrm{m}$ from Agilent. Mobile phases $\mathrm{A}$ and $\mathrm{B}$ were water and acetonitrile, respectively, both containing $0.1 \%$ of formic acid. Fatty acids separation was carried out by the following gradient program: 62\% B (held for $4.5 \mathrm{~min}$ ) followed by a linear increase up to $100 \% \mathrm{~B}$ in $10 \mathrm{~min}$ (held for $1 \mathrm{~min}$ ). The column temperature was set at $45^{\circ} \mathrm{C}$ and the injection volume was $2 \mu \mathrm{l}$.

Mass detection was performed using a Bruker Impact II q-ToF mass spectrometer with electrospray ionization, operated in the negative mode. The settings of the mass spectrometer were as follows: spray voltage, $4.5 \mathrm{kV}$; drying gas flow $12 \mathrm{l} / \mathrm{min}$; drying gas temperature $250^{\circ} \mathrm{C}$; nebulizer pressure $44^{\circ} \mathrm{psi}$.

For quantitation, calibration curves for each compound were prepared by proper dissolution of the pure standards in methanol to encompass the expected concentration of the analytes in the sample. The calibration ranges were as follows: $0.4-12.5 \mu \mathrm{g} / \mathrm{ml}$ for eicosapentaenoic (EPA) and $\gamma$-linolenic; $1.2-37.5 \mu \mathrm{g} / \mathrm{ml}$ for docosahexaenoic acid (DHA) and linolenic; 2.3-75 $\mu \mathrm{g} / \mathrm{ml}$ for arachidonic (AA) and palmitoleic; 3.9-125 $\mu \mathrm{g} / \mathrm{ml}$ for linoleic; and $7.8-250 \mu \mathrm{g} / \mathrm{ml}$ for oleic, palmitic, and stearic. A good linearity was observed in all cases $\left(r^{2}>0.994\right)$. Heptadecanoic acid, added at a level of $30 \mu \mathrm{g} / \mathrm{ml}$, was used as internal standard to compensate for possible biases during the sample preparation step.

\section{Quantification of Cytokines}

Serum levels of IFN $\gamma$, MCP-1, and IL- 6 were determined by immunoassays using commercial kits (IFN $\gamma$ OptEIA kit) from BD Biosciences (NJ, USA) and MCP-1 and IL-6 mini-EDK kits from Peprotech (NJ, USA), following manufacturer's instructions. Detection limits were $0.58,8$, and $5.8 \mathrm{pg} / \mathrm{ml}$ for IFN $\gamma$, MCP-1, and IL-6, respectively.

\section{Nutritional Assessments}

Dietary intake was assessed by means of an annual semi quantitative validated food frequency questionnaire including 160 items (25). Trained dieticians asked about cooking practices, number and amount of ingredients used in each recipe, as well as enquiring about menu preparation (e.g., type of oil used and type of milk) and other relevant information to the study. During an interview, subjects were asked item by item whether they usually ate each food and, if so, how much they usually ate. For this purpose, three different serving sizes of each cooked food were presented in pictures to the participants so that they could choose from up to seven serving sizes (from "less than the small one" to "more than the large one"). For some of the foods consumed, amounts were recorded in household units, by volume, or by measuring with a ruler. Methodological issues concerning dietary assessment have been detailed elsewhere (25). Food intake was analyzed for energy, macronutrients, and total fiber content by using the nutrient Food Composition Tables developed by the Centro de Enseñanza Superior de Nutrición Humana y Dietética (26).

\section{Anthropometric Measures}

Height was measured using a stadiometer with an accuracy of $\pm 1 \mathrm{~mm}$ (Año-Sayol, Barcelona, Spain). The subjects stood barefoot, in an upright position and with the head positioned in the Frankfort horizontal plane. Weight was measured on a scale with an accuracy of $\pm 100 \mathrm{~g}$ (Seca, Hamburg, Germany). Quetelet index was calculated using the formula: weight $(\mathrm{kg}) /$ height $\left(\mathrm{m}^{2}\right)$.

\section{Analysis of Fecal Microbiota}

Fecal samples from individuals participating in the study were collected at home in sterile containers, kept at $4^{\circ} \mathrm{C}$ in the domestic refrigerator (maximum $2-3 \mathrm{~h}$ from deposition) and frozen at $-80^{\circ} \mathrm{C}$ until analyses just right on arrival to the laboratory, as previously reported $(27,28)$. One gram of fecal sample was employed for DNA extraction by using the QIAamp DNA stool mini kit (Qiagen, Hilden, Germany) as previously described (28). Quantification of bacterial groups (Akkermansia, Bacteroides group, Bifidobacterium, Clostridium cluster XIVa, Lactobacillus group, and Faecalibacterium) in feces was performed with a 7500 Fast Real Time PCR System (Applied Biosystems, Foster 
City, CA, USA) using SYBR Green PCR Master Mix (Applied Biosystems) as previously described (29-31). These microbial groups include the main representatives of the human intestinal microbiota, which all together represent over $95 \%$ of the total bacteria in the human intestine $(32,33)$. For qPCR analysis, $1 \mu \mathrm{l}$ of template fecal DNA ( $5 \mathrm{ng})$ and $0.2 \mu \mathrm{M}$ of each primer were added to the reaction mixture $(25 \mu \mathrm{l})$. PCR cycling was as follows: an initial cycle of $95^{\circ} \mathrm{C} 10 \mathrm{~min}, 40$ cycles of $95^{\circ} \mathrm{C} 15 \mathrm{~s}$, and $1 \mathrm{~min}$ at the appropriate primer temperature. We compared the $C_{\mathrm{t}}$ values obtained from a standard curve constructed as previously indicated (30) to estimate the number of cells. Standard cultures, primers, and annealing temperatures used for qPCR were the same as those recently reported (31).

\section{Analysis of Short-Chain Fatty Acids in Fecal Samples}

Analysis of short chain fatty acids (SCFA) was performed by gas chromatography to determine the concentrations of acetate, propionate, and butyrate. One gram of fecal samples was weighed, diluted 1:10 in sterile PBS, and homogenized in a LabBlender 400 stomacher (Seward Medical, London, UK) at full speed for $4 \mathrm{~min}$. Supernatants were then obtained by centrifugation $\left(10,000 \mathrm{~g}, 30 \mathrm{~min}, 4^{\circ} \mathrm{C}\right)$, filtered through $0.2-\mu \mathrm{m}$ filters, mixed with $1 / 10$ of ethyl butyric $(2 \mathrm{mg} / \mathrm{ml})$ as an internal standard, and stored at $-80^{\circ} \mathrm{C}$ until analysis. A gas chromatograph $6890 \mathrm{~N}$ (Agilent Technologies Inc., Palo Alto, CA, USA) connected to a mass spectrometry (MS) 5973N detector (Agilent Technologies) and to a flame ionization detector was used for identification and quantification of SCFA.

Data were collected using the Enhanced ChemStation G1701DA software (Agilent). Samples $(1 \mu \mathrm{l})$ were directly injected into the gas chromatograph equipped with an HP-Innowax capillary column (60-m length by $0.25-\mathrm{mm}$ internal diameter, with a $0.25 \mu \mathrm{m}$ film thickness; Agilent) using $\mathrm{He}$ as gas carrier and a constant flow rate of $1.5 \mathrm{ml} / \mathrm{min}$. The temperature of the injector was kept at $220^{\circ} \mathrm{C}$, and the split ratio was 50:1. Chromatographic conditions were as follows: initial oven temperature of $120^{\circ} \mathrm{C}$, $5^{\circ} \mathrm{C} / \mathrm{min}$ up to $180^{\circ} \mathrm{C}, 1 \mathrm{~min}$ at $180^{\circ} \mathrm{C}$, and a ramp of $20^{\circ} \mathrm{C} / \mathrm{min}$ up to $220^{\circ} \mathrm{C}$ to clean the column. In the MS detector, the electron impact energy was set at $70 \mathrm{eV}$. The data collected were in the range of 25-250 atomic mass units (at 3.25 scans/s).

SCFA were identified by comparison of their mass spectra with those held in the HP-Wiley 138 library (Agilent) and by comparison of their retention times with those of the corresponding standards (Sigma Aldrich, St. Louis, MO, USA). The peaks were quantified as relative abundances with respect to the internal standard. The concentration (in $\mathrm{mM}$ ) of each SCFA was calculated using the linear regression equations $\left(r^{2} \geq 0.99\right)$ from the corresponding standard curves obtained with six different concentrations.

\section{Statistical Analysis}

The study design was a cross-sectional, one-group, observational analysis. Continuous variables were summarized as median (interquartile range) or mean $\pm \mathrm{SD}$, whereas $n$ (\%) was used for the categorical ones. Mann-Whitney $U$ or $t$-tests were used to assess statistical differences, when appropriate. Correlations were analyzed by Spearman's rank test. After univariate correlation analyses, multiple regression analyses were conducted to assess the strength of the association between these variables including other (continuous) variables as potential confounders. Multiple regression analyses were also performed to identify the main predictors of a candidate independent variable. When coefficients of determination correspond to multiple regression analyses they are indicated as $\mathrm{R}^{2}$. For each analysis, dependent and independent variables were indicated and the $\beta$ coefficient, $B$ coefficient with $95 \%$ confidence intervals (CIs), and $p$-values were computed. The association between two categorical variables was first studied by $\chi^{2}$ tests in univariate models and then was analyzed by multiple logistic regression models to include potential confounders as independent variables. For this analysis, odds ratio (OR) and 95\% CI were computed. Principal component analysis (PCA) with Varimax rotation was performed to reduce sample dimensionality and potential collinearity effects. The number of components retained was based on eigenvalues $(>1)$, and loadings greater than 0.5 were used to identify the variables comprising a single component. For cluster analysis, squared Euclidean distances were calculated, and Ward's Minimum Variance Method was used to identify clusters minimizing the loss of information. $\mathrm{R}$ package heatmap. 2 was used to generate heatmaps for visualization purposes. Some statistical analyses were performed independently in each cluster to evaluate whether differences among the studied variables independently arise on each cluster. The statistical approach is summarized in Figure 1. SPSS 19.0, R 3.0.3, and GraphPad Prism 5.0 for Windows were used.

\section{RESULTS}

\section{FFA and Microbial Populations}

Total FFA serum levels and fecal microbial groups were analyzed in 101 human adults from the general population (Table 1). High total FFA serum levels were considered as a surrogate marker of impaired lipid metabolism. Then, the associations between FFA levels and intestinal microbial populations were determined by univariate and multivariate analyses adjusted for socio-demographical [age, gender, and body mass index (BMI)] and nutritional parameters (total energy and intakes of carbohydrates, lipids, proteins, and fiber). Interestingly, only Akkermansia abundance was correlated with FFA serum levels $(r=-0.383, p<0.001)$ (Figure 2). Moreover, this association remained statistically significant in a multiple regression analysis adjusted for age, gender, BMI, microbial groups analyzed, total energy as well as carbohydrates, lipids, and proteins intake as potential confounders (Table 2). Although a slight correlation between total FFA and the serum levels of the pro-inflammatory cytokine IL- 6 was observed ( $r=0.240, p=0.020$ ), no effect was evidenced when IL- 6 was introduced in the previous multiple regression analysis.

Next, we aimed to evaluate whether this finding was due to a general effect on total FFA levels or it could be also associated with individual FFA species. To this end, associations between Akkermansia and the levels of individual FFA ( $\gamma$-linolenic, palmitic, oleic, stearic, linoleic, palmitoleic, linoleic, AA, EPA, and 


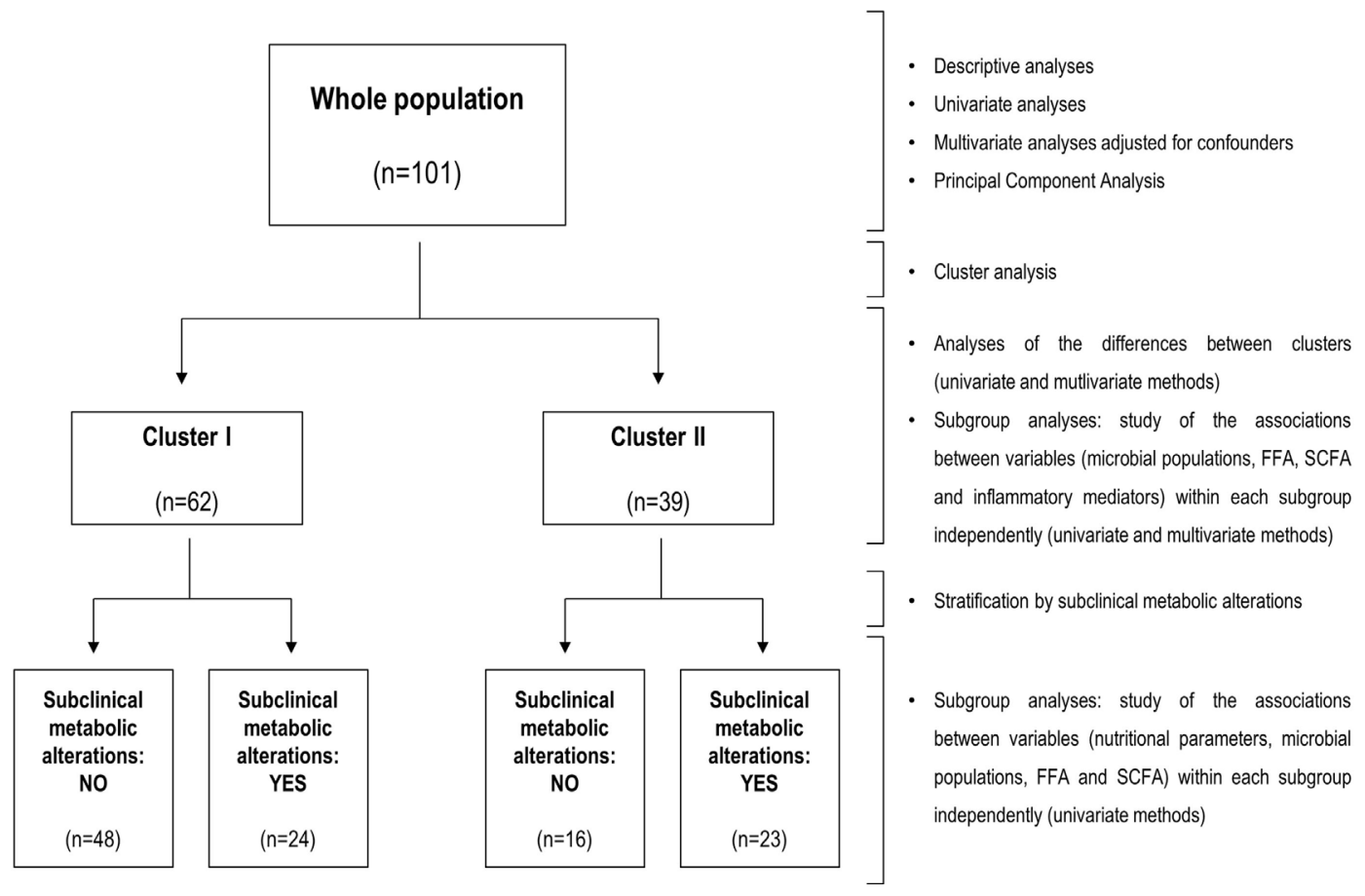

FIGURE 1 | Flow diagram of the statistical analyses. Summary of the workflow followed in the statistical analyses performed in the present study. The flow diagram (left) indicates groups analyzed and sample sizes, whereas statistical methods for analysis and stratification of the study population are indicated at the left side.

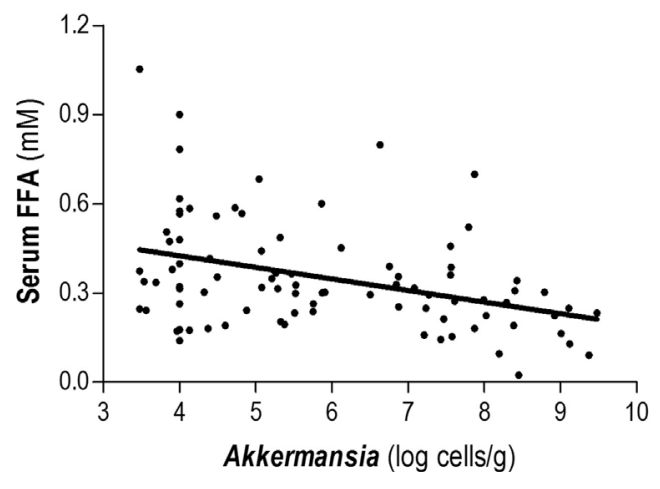

FIGURE 2 | Association between Akkermansia and serum total free fatty acids (FFA) levels. Abundance of Akkermansia in fecal samples was negatively correlated with the total levels of serum FFA in healthy subjects. Correlation was assessed by Spearman rank's test.

DHA) were assessed. Akkermansia abundance was negatively associated with some FFA [stearic $(r=-0.218, p=0.039)$, palmitic $(r=-0.321, p=0.002)$, oleic $(r=-0.261, p=0.013)$, palmitoleic $(r=-0.297, p=0.004)$, linoleic $(r=-0.272, p=0.010)$, and $\gamma$-linolenic $(r=-0.232, p=0.028)]$, but no association was observed with EPA, DHA, AA, or linoleic levels. As important differences among FFA exist, individual FFA were grouped according to their chemical structure: saturated (SFA), monounsaturated (MUFA), w3-poly-unsaturated (w3-PUFA), and w6-PUFA. Interestingly, Akkermansia exhibited a strong negative association with SFA $(r=-0.314, p=0.003)$ as well as with
TABLE 2 | Akkermansia as predictor of free fatty acids (FFA) serum levels.

\begin{tabular}{lrcr}
\hline & $\boldsymbol{\beta}$ & $\boldsymbol{B}[\mathbf{9 5 \%} \mathbf{~ C l}]$ & $\boldsymbol{p}$-Value \\
\hline Age & -0.099 & $-0.001[-0.006,0.003]$ & 0.515 \\
Gender & -0.119 & $-0.045[-0.131,0.042]$ & 0.309 \\
BMI & 0.078 & $0.003[-0.006,0.012]$ & 0.463 \\
Akkermansia & -0.350 & $-0.035[-0.055,-0.014]$ & $<\mathbf{0 . 0 0 1}$ \\
Bacteroides group & -0.080 & $-0.013[-0.055,0.029]$ & 0.550 \\
Bifidobacterium & -0.041 & $-0.008[-0.055,0.039]$ & 0.742 \\
Clostridium cluster XIVa & -0.064 & $-0.008[-0.041,0.025]$ & 0.627 \\
Lactobacillus group & 0.180 & $0.031[-0.013,0.074]$ & 0.169 \\
Faecalibacterium & 0.092 & $0.015[-0.038,0.067]$ & 0.584 \\
Total energy & 0.141 & $0.001[-0.001,0.002]$ & 0.141 \\
Carbohydrates intake & 0.604 & $0.002[-0.001,0.004]$ & 0.160 \\
Lipids intake & 0.606 & $0.004[-0.001,0.009]$ & 0.146 \\
Proteins intake & 0.173 & $0.001[-0.002,0.005]$ & 0.510
\end{tabular}

The associations between FFA serum levels (as dependent variable) and microbial populations were studied by multiple lineal regression analysis including sociodemographical parameters and nutritional intakes as potential confounders (all included as independent variables in the model). $\beta$ and B coefficients (with 95\% confidence intervals $(\mathrm{Cl})]$ and $p$-values are calculated for each parameter within the same model. $R^{2}$ (model) $=0.303$. The $p$-value highlighted in bold represent statistically significant differentes.

MUFA and w6-PUFA to a lower degree $(r=-0.266, p=0.011$ and $r=-0.244, p=0.020$, respectively), whereas no association was observed for w3-PUFA $(r=-0.104, p=0.330)$. Thus, our findings point to a negative association between Akkermansia abundance and levels of SFA, MUFA, and w6-PUFA, all of them pro-inflammatory. Since some collinearity was noted among individual FFA levels, a PCA was carried out to avoid potential biases. PCA was then conducted with the serum levels of specific 
FFA, given that all of them exhibited communalities higher than 0.5 , thus supporting the appropriateness of this analysis. The Kaiser-Meyer-Olkin test provided a good adequacy of the data $(0.858)$ as the Bartlett test of sphericity $\left(p=10^{-144}\right)$ did. PCA identified two main components (explaining $72.4 \%$ of the total variance): C1 (including loadings from $\gamma$-linolenic, palmitic, oleic, stearic, linoleic, palmitoleic, linoleic, AA; explaining $59.81 \%$ of the total variance) and C2 (including EPA and DHA; explaining $13.09 \%$ of the total variance). Notably, Akkermansia was negatively associated with $\mathrm{C} 1(r=-0.308, p=0.003)$, thus supporting an opposite relationship between the levels of this microorganism and those of the saturated, monounsaturated, and w6-PUFA retained in this component, whereas no association was found with anti-inflammatory w3-PUFA (C2). These results from the PCA strengthen our previous findings on the separated analysis of FFA according to their chemical structure. Moreover, since PUFA are present in lower levels, and taking into account that nine FFA loaded on $\mathrm{C} 1$ whereas only two did in $\mathrm{C} 2$, this association was studied by regression analyses including total FFA serum levels as confounder to rule out the possibility that decreased FFA abundance may bias the analysis. Interestingly, Akkermansia levels remained associated with $\mathrm{C} 1$ after performing this adjustment $(\beta[95 \% \mathrm{CI}], p$ : $-0.286[-1.999,-0.315]$, $p=0.008)$, whereas no association was observed with C2 $(-0.052$ $[-0.152,-0.092], p=0.625)$, thereby confirming our previous findings. In addition, the analysis of inflammatory mediators showed that Akkermansia was negatively associated with IL-6 serum levels $(r=-0.233, p=0.032)$, whereas IL-6 levels and C1 were in turn associated $(r=0.220, p=0.032)$. No associations were found for Akkermansia with IFN $\gamma(r=0.056, p=0.604)$ or MCP-1 $(r=-0.028, p=0.802)$ serum levels.

Therefore, all these results suggest a relationship between intestinal Akkermansia and serum FFA levels in humans. Moreover, Akkermansia was negatively associated with specific FFA species, mainly those saturated and/or with an attributed pro-inflammatory role, such as MUFA and w6-PUFA. This finding is in line with the negative association observed between Akkermansia and IL-6 serum levels.

\section{Associations between FFA Profiles, Microbial Populations, and SCFA}

Our results from the PCA analysis and the differences found in the associations with Akkermansia and IL- 6 levels point to the existence of different FFA profiles among individuals. To address this hypothesis, an unsupervised cluster analysis was performed for the individual FFA levels. Interestingly, this approach confirmed the existence of two independent groups of individuals based on FFA levels, thereafter referred to as cluster I $(n=62)$ and cluster II ( $n=39)$ (Figure 3A).

Although all FFA species were higher in cluster II, mainly saturated and/or pro-inflammatory fatty acids were associated with this group, whereas a strikingly different pattern was observed for EPA and DHA, which seem not to play a role in clusters definition on our analysis (Figure 3B). No significant differences in age, gender or BMI were observed between individuals from clusters I and II (Table 3). Nutrient intakes were then compared between clusters in order to evaluate whether distinct daily intakes could account for the differences observed in FFA clusters. Interestingly, no differences in daily intakes were found (Table 4), hence supporting that additional factors other than demographic and nutritional parameters may underlie the differences found between FFA clusters.

Notably, important differences in intestinal microbial populations and fecal SCFA levels were observed between clusters (Table 5). Cluster II was characterized by decreased levels of Akkermansia and increased Lactobacillus group counts as well as higher acetate, propionate and total fecal SCFA concentrations than cluster I. Moreover, increased total FFA [0.48 (0.24) vs 0.26 (0.13) $\mathrm{mM}]$ and IL-6 [752.40 (1,155.22) vs $411.77(636.85) \mathrm{pg} / \mathrm{ml}]$, but not IFN $\gamma(p=0.252)$ or MCP-1 $(p=0.541)$ serum levels were also registered in cluster II (Figure 3C).

Finally, some statistical analyses performed independently within each cluster revealed particular interesting features for each group, thereby stressing the existence of important differences between groups. On the one hand, IL-6 serum levels were negatively correlated to Akkermansia abundance $(r=-0.312$, $p=0.034)$ and acetate $(r=-0.344, p=0.014)$ in subjects included in cluster I, whereas no associations were observed in cluster II, characterized by higher IL- 6 and acetate levels. Conversely, acetate and total fecal SCFA were strongly associated with higher total FFA serum levels in subjects included in cluster II ( $r=0.459, p=0.006$; and $r=0.410, p=0.016$, respectively). Similarly, total energy intake was positively associated in this group with total SCFA production $(r=0.438, p=0.008)$, but not in their cluster I counterparts $(r=-0.044, p=0.748)$, hence supporting a cross talk between host and microbial metabolisms, in which SCFA are involved.

Overall, our findings support the existence of homeostatic relationship between microbial populations, host lipid metabolism and inflammatory mediators. Thus, imbalanced microbial populations and/or increased fecal SCFA production could be related to an altered lipid metabolism as well as a serum IL-6 shift.

\section{Analysis of the Associations among Impaired FFA Profile, Subclinical Metabolic Alterations, and Microbial Populations}

Then, we addressed in our group of adult subjects, whether an altered gut microbiota composition and/or serum inflammatory mediators may have a negative impact on the host metabolism and if a clinical relevance of these players could be expected.

Although no significant differences in serum levels of glucose, cholesterol or triglycerides were found between groups, individuals within cluster II were more likely to exhibit hyperglycemic $(>100 \mathrm{mg} / \mathrm{dl})$ or hypertriglycemic $(>150 \mathrm{mg} / \mathrm{dl})$ states than those within cluster I $(22 / 39$ vs $22 / 62, p=0.039$ and $7 / 39$ vs $4 / 62, p=0.070$ ). Consequently, metabolic alterations (either hyperglycemia or hypertriglycemia, as stated in the Methods section) were more prevalent in cluster II (23/39 vs $24 / 62$, $p=0.047)$.

The strength of these associations was further analyzed by multiple logistic regression. By selecting subclinical metabolic 


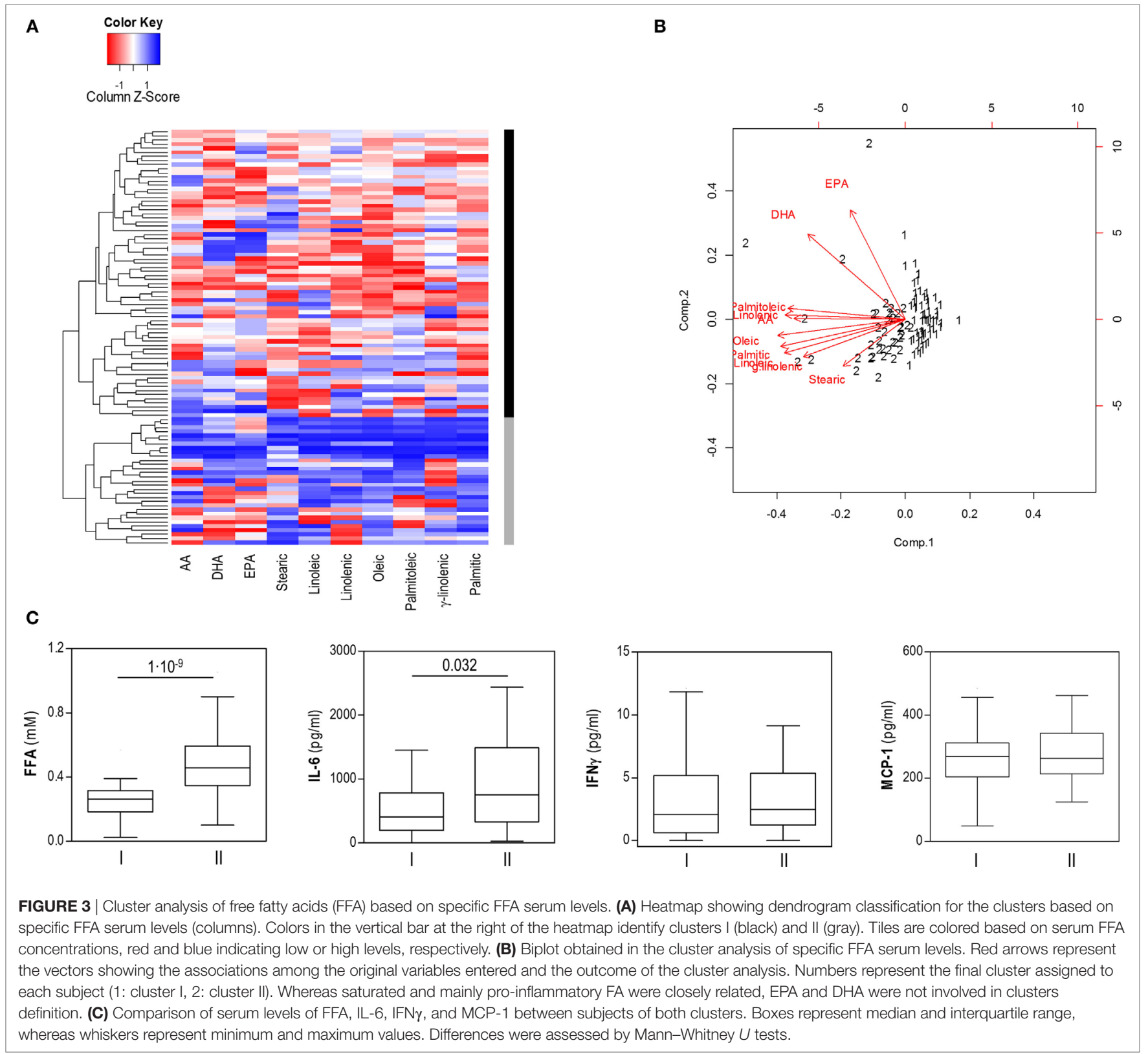

TABLE 3 | Demographical parameters according to free fatty acids (FFA) clusters.

\begin{tabular}{lcccc}
\hline & $\begin{array}{c}\text { Study population } \\
(\boldsymbol{n}=\mathbf{1 0 1})\end{array}$ & $\begin{array}{c}\text { Cluster I } \\
(\boldsymbol{n}=\mathbf{6 2})\end{array}$ & $\begin{array}{c}\text { Cluster II } \\
(\boldsymbol{n}=\mathbf{3 9})\end{array}$ & $\boldsymbol{p}$-Value \\
\hline $\begin{array}{l}\text { Demographical parameters } \\
\text { Age, mean } \\
\text { (range) }\end{array}$ & $49.41(19-72)$ & $50.39(25-72)$ & $47.78(19-67)$ & 0.260 \\
$\begin{array}{l}\text { Gender (F/M) } \\
\text { BMl }\end{array}$ & $66 / 35$ & $42 / 20$ & $24 / 15$ & 0.524 \\
\hline
\end{tabular}

Demographical parameters from the whole population $(n=101)$ and from the study participants classified according to clusters computed from individual FFA levels are shown. Differences between clusters were analyzed by Mann-Whitney $U$ or $\chi^{2}$ tests. Variables are expressed as mean $\pm S D$, median (interquartile range) or $n$, as appropriate. alterations as the dependent variable and adjusting the model for confounders (age, gender, BMI, total energy, as well as lipids, carbohydrates, protein, and fiber intakes, all introduced as independent variables), we found that individuals within cluster II were more likely to exhibit metabolic alterations (OR [95\% $\mathrm{CI}], p: 3.064$ [1.127, 8.330], 0.028). Interestingly, this association remained significant when the microbial groups were entered in the model, appearing Akkermansia abundance also associated with metabolic alterations $(p=0.029)$. This highlights the interplay between altered FFA profile, microbial populations and impaired host metabolism.

Moreover, additional observations reinforced the finding commented just above. Since our initial results point to a cross 
TABLE 4 | Nutritional parameters according to free fatty acids (FFA) clusters.

\begin{tabular}{|c|c|c|c|c|c|}
\hline & Study population $(n=101)$ & Cluster I $(n=62)$ & Cluster II $(n=39)$ & $p$-Value (unadjusted) & $p$-Value (adjusted) \\
\hline \multicolumn{6}{|l|}{ Daily intakes } \\
\hline Energy (kcal) & $1,993.24 \pm 531.62$ & $1,961.40 \pm 536.41$ & $2,011.51 \pm 515.05$ & 0.660 & 0.842 \\
\hline Lipids (g) & $80.49 \pm 27.34$ & $80.64 \pm 26.31$ & $78.23 \pm 27.77$ & 0.494 & 0.126 \\
\hline Saturated (g) & $26.24 \pm 10.00$ & $26.82 \pm 10.82$ & $25.04 \pm 8.54$ & 0.512 & 0.109 \\
\hline Monounsaturated (g) & $33.83 \pm 13.25$ & $35.08 \pm 12.82$ & $30.68 \pm 11.86$ & 0.100 & 0.079 \\
\hline Polyunsaturated (g) & $13.94 \pm 9.06$ & $12.41 \pm 6.24$ & $15.91 \pm 11.96$ & 0.410 & 0.138 \\
\hline Proteins (g) & $92.15 \pm 27.66$ & $93.36 \pm 28.79$ & $88.76 \pm 25.58$ & 0.754 & 0.132 \\
\hline Carbohydrates (g) & $213.06 \pm 69.43$ & $207.73 \pm 66.70$ & $226.79 \pm 73.77$ & 0.125 & 0.070 \\
\hline Fiber (g) & $22.71 \pm 10.65$ & $23.46 \pm 11.74$ & $21.75 \pm 8.88$ & 0.532 & 0.249 \\
\hline Soluble (g) & $2.75 \pm 1.21$ & $2.79 \pm 1.16$ & $2.74 \pm 1.32$ & 0.551 & 0.972 \\
\hline Insoluble (g) & $14.13 \pm 5.88$ & $14.50 \pm 6.11$ & $13.78 \pm 5.57$ & 0.523 & 0.545 \\
\hline
\end{tabular}

Dietary intakes from the whole population $(n=101)$ and from the study participants classified according to clusters computed from individual FFA levels are shown. Differences in daily intakes between clusters were analyzed by Mann-Whitney $U$ tests (unadjusted) or linear regression models to adjust for confounders (adjusted). Energy was adjusted by gender and age, whereas the rest of the nutrients were adjusted by gender, age, and energy. Variables are expressed as mean $\pm S D$.

TABLE 5 | Intestinal microbial populations, fecal short-chain fatty acid (SCFA), and serum cytokine levels according to free fatty acids (FFA) clusters.

$\begin{array}{cccc}\begin{array}{c}\text { Study } \\ \text { population } \\ (n=101)\end{array} & \begin{array}{c}\text { Cluster I } \\ (n=62)\end{array} & \begin{array}{c}\text { Cluster II } \\ (n=39)\end{array} & p \text {-Value } \\ & & & \end{array}$

\begin{tabular}{|c|c|c|c|c|}
\hline \multicolumn{5}{|c|}{ Microbial populations (log cells/g) } \\
\hline Akkermansia & $5.84 \pm 1.80$ & $6.24 \pm 1.84$ & $5.09 \pm 1.47$ & 0.002 \\
\hline Bacteroides group & $9.15 \pm 1.16$ & $9.05 \pm 1.21$ & $9.19 \pm 1.17$ & 0.587 \\
\hline Bifidobacterium & $8.26 \pm 0.91$ & $8.02 \pm 1.44$ & $8.35 \pm 0.95$ & 0.233 \\
\hline $\begin{array}{l}\text { Clostridium cluster } \\
\text { XIVa }\end{array}$ & $8.52 \pm 1.41$ & $8.30 \pm 1.52$ & $8.63 \pm 1.37$ & 0.306 \\
\hline $\begin{array}{l}\text { Lactobacillus } \\
\text { group }\end{array}$ & $6.14 \pm 1.04$ & $5.92 \pm 1.14$ & $6.41 \pm 0.86$ & 0.024 \\
\hline Faecalibacterium & $8.05 \pm 1.29$ & $7.84 \pm 1.15$ & $8.24 \pm 1.11$ & 0.105 \\
\hline \multicolumn{5}{|l|}{ SCFA (mM) } \\
\hline Acetate & $40.46 \pm 19.20$ & $35.43 \pm 14.32$ & $49.31 \pm 22.98$ & 0.001 \\
\hline Propionate & $15.04 \pm 8.34$ & $13.03 \pm 6.01$ & $18.26 \pm 10.45$ & 0.003 \\
\hline Butyrate & $10.61 \pm 7.19$ & $9.74 \pm 7.07$ & $12.08 \pm 7.31$ & 0.135 \\
\hline Total SCFA & $66.42 \pm 31.06$ & $58.21 \pm 23.75$ & $79.66 \pm 36.95$ & 0.004 \\
\hline \multicolumn{5}{|c|}{ Cytokine levels (pg/ml) } \\
\hline IL-6 & $\begin{array}{l}442.79 \\
(949.99)\end{array}$ & $\begin{array}{l}411.72 \\
(636.85)\end{array}$ & $\begin{array}{l}752.40 \\
(1,155.22)\end{array}$ & 0.032 \\
\hline MCP-1 & $\begin{array}{l}267.30 \\
(127.83)\end{array}$ & $\begin{array}{l}268.48 \\
(101.13)\end{array}$ & $\begin{array}{l}263.20 \\
(128.51)\end{array}$ & 0.541 \\
\hline $\mathrm{IFN} \gamma$ & $2.05(4.70)$ & $2.05(4.64)$ & $2.48(4.12)$ & 0.252 \\
\hline
\end{tabular}

Microbial populations and SCFA fecal levels and serum cytokines from the whole population ( $n=101$ ) and from the study participants classified according to clusters computed from individual FFA levels are shown. Differences in microbial populations, fecal SCFA, and serum cytokine levels between clusters were analyzed by t or MannWhitney $U$ tests. The $p$-values highlighted in bold represent statistically significant differences. Variables are expressed as mean $\pm S D$ or median (interquartile range).

talk between host and microbial metabolism, we further deepen into this idea focusing on possible differential associations between microbial groups or SCFA with nutrient intakes. In subjects with subclinical metabolic alterations, Lactobacillus abundance was correlated with carbohydrates intake in both cluster I $(r=0.603, p=0.004)$ and II $(r=0.765, p<0.001)$, but with fiber intakes only in cluster I $(r=0.609, p=0.003)$. No associations were observed in those subjects free of metabolic alterations. On the other hand, the association between total SCFA and total FFA levels mirrored this situation, a positive correlation being found in individuals with metabolic alterations in both cluster I $(r=0.524, p=0.018)$ and II $(r=0.432, p=0.045)$, but not in those individuals without subclinical metabolic alterations.

In sum, these results support an association between microbial populations, nutritional factors, and impaired host metabolism. Subjects exhibiting an altered FFA profile, which also display imbalanced Akkermansia and Lactobacillus populations, were more frequently associated with subclinical pathogenic metabolic states. Divergent patterns in the associations between dietary intakes with microbial populations and SCFA were also observed.

\section{Microbial Imbalanced Populations As Predictors of Impaired FFA Levels}

Finally, although Akkermansia abundance was identified as the only microbial predictor of serum FFA levels in the whole population analyzed $(n=101)$, striking differences in Akkermansia levels were evidenced between clusters I and II. Since the results commented just above suggest that altered microbial populations and their interactions with nutrient intakes may underlie impairment in the host metabolism and that SCFA can have relevance in such interactions, we included all these parameters (microbial populations, SCFA, dietary intakes, age, gender, and $\mathrm{BMI}$ ) as independent variables in a multivariate regression analysis, FFA levels being selected as the dependent variable, after stratifying our populations by the clusters obtained in the previous analysis.

Interestingly, whereas only age $(p=0.038)$ and BMI ( $p=0.050)$ were identified as predictors of FFA levels in individuals within cluster I $\left(R^{2}(\right.$ model $\left.)=0.407\right)$, Lactobacillus abundance $(p=0.032)$, fiber intake $(p=0.021)$, SCFA (acetate $p=0.008$, propionate $p=0.018$, and butyrate $p=0.003$ ), and gender $(p=0.008)$ were associated with FFA levels in subjects within cluster II $\left(R^{2}(\right.$ model $\left.)=0.646\right)$.

In summary, different factors were found to be associated with total FFA levels depending on the levels of Akkermansia. Above a threshold of Akkermansia levels, anthropometric factors such as BMI and age were the main predictors of serum FFA. However, when Akkermansia abundance is low, factors other than the anthropometric characteristics (as imbalanced microbial populations or SCFA production) seem to impact the FFA pool. 


\section{DISCUSSION}

Despite the important research advances in recent years, the links between human metabolism and gut microbiota are far from being completely understood, especially in the field concerning the lipid metabolism. The present study addresses a multilevel analysis of this scenario, by assessing different surrogate biomarkers of the lipid metabolism, in addition to some relevant intestinal microbial groups and SCFA production as well as inflammatory mediators; the study was performed in a sample of adult subjects from the general population in order to gain some insight into the relationship among these parameters and their potential impact on the human health. Our results revealed an association between the abundance of the intestinal microorganism Akkermansia and circulating FFA. This association was restricted to a specific group of FFA species, Akkermansia and serum IL-6. In addition, cluster analyses revealed that imbalanced intestinal microbial groups and levels of SCFA production may be related to an impaired FFA profile, pro-inflammatory and saturated fatty acids mainly hallmarking this group. Interestingly, individuals who exhibit these features were more likely to show metabolic alterations. Therefore, the results herein presented provide valuable information on the gut microbiota-host metabolism axis and its involvement in human health.

A major finding of our study was the association between Akkermansia and serum FFA levels. Akkermansia has been reported to participate in the maintenance of gut integrity and energy harvest by the host (34). It has been confirmed that Akkermansia has a causative role in lowering body fat mass and in glucose homeostasis in mice models $(35,36)$, although evidence in humans is limited. Our results demonstrate for the first time an association between Akkermansia and serum FFA in a cohort of human adults from the general population. Interestingly, we were able to cluster these healthy adults into two independent groups on the basis of serum individual FFA species, one of these groups showing an increased prevalence of subclinical metabolic alterations. In our study Akkermansia was negatively associated mainly with saturated FFA, in line with the differences that have been previously reported by other authors between lard-like and fish oil-enriched diets in the gut microbiota of mice $(36,37)$. Moreover, differences in Akkermansia abundance found in our study between FFA clusters were associated in turn with striking differences in total FFA levels. This observation supports a gradual relationship between Akkermansia abundance and FFA serum levels and therefore aligns with the concept proposed by other authors $(36,38)$ that a "threshold" for Akkermansia levels may exist. In line with this hypothesis, below certain levels of Akkermansia abundance, gut barrier, and other functions developed by this microbe may become insufficient, thus promoting a shift from a healthy toward a pathologic-prone status. As previously commented, different parameters were found to be predictors of total FFA serum levels depending on the Akkermansia levels. Therefore, low Akkermansia levels may render the host metabolism more sensitive to a number of factors which can lead to an imbalanced FFA profile and, potentially, altered metabolism, hence supporting this notion.
Akkermansia is known to reside within the mucus layer of the intestine, thus contributing to strengthen the intestinal wall (34). Then, it is feasible that decreased Akkermansia levels may lead to a compromised barrier function and increased gut permeability, hence promoting metabolic endotoxemia, which has been related to the development of obesity and associated disorders $(35,39)$. Interestingly, we have found that decreased Akkermansia levels were associated with elevated IL-6 serum concentrations and impaired FFA profile, and the subpopulation with such profile exhibited a bias toward the enrichment in pro-inflammatory and saturated fatty acids, as well as an increased prevalence of metabolic disturbances, thus supporting those previous findings. Therefore, these results suggest that Akkermansia may be linked to the inflammatory milieu by modulating the FFA profile in the host.

Previous studies have revealed reduced levels of Akkermansia in patients suffering from inflammatory bowel disease or other metabolic impaired conditions $(40,41)$, although some controversy exist on the role of Akkermansia in these disorders. Interestingly, a recent study reported decreased counts of Akkermansia in pre-diabetic individuals (42), suggesting an early involvement of this microorganism in metabolic disorders. Thus, we decided to focus on subclinical metabolic features (suggestive of an impaired metabolism), which can be detected in healthy subjects, in order to improve our knowledge on the potential role of Akkermansia as an early marker of impaired metabolic conditions in the general population. These metabolic features were related to an increased risk of metabolic complications in the long-term (43-48). Thus, these subjects showing such subclinical alterations could be classified as "at risk" population according to the scientific literature. Based on this approach, it may be hypothesized that imbalanced microbial populations can underlie the subclinical stage of some metabolic conditions. Actually, the lack of association in our study between Akkermansia and other clinical hard end-points, such as obesity or related immune mediators, such as MCP-1 or IFN $\gamma$, may support a very early role of these features. However, long-term studies are warranted.

Importantly, we found an opposite behavioral pattern between Akkermansia and Lactobacillus groups, the latter being overrepresented in subjects with impaired FFA profile and increased prevalence of metabolic traits. Although classically regarded as "beneficial" microbes, some recent evidence highlights a positive association between Lactobacillus and BMI $(49,50)$ as well as with some pathological outcomes (51-53). In fact, a slight increase in the relative abundance of Lactobacillales has been reported in the context of the inflammatory condition of Behçet syndrome (54), related to an altered SCFA production and aberrant immune responses. Thus, these pieces of evidence point to a Lactobacillus within-group heterogeneity with relevance for the human health, as suggested by other authors $(40,55)$. In agreement with this hypothesis our findings on the interactions between Lactobacillus abundance and nutrient parameters, evidenced different association patterns as depending on the metabolic "health" status of the subject. Controversy observed in mice studies and clinical interventions in humans is also consistent with this idea. A recent work shows opposite patterns upon mucin usage as substrate by Lactobacillus and Akkermansia 


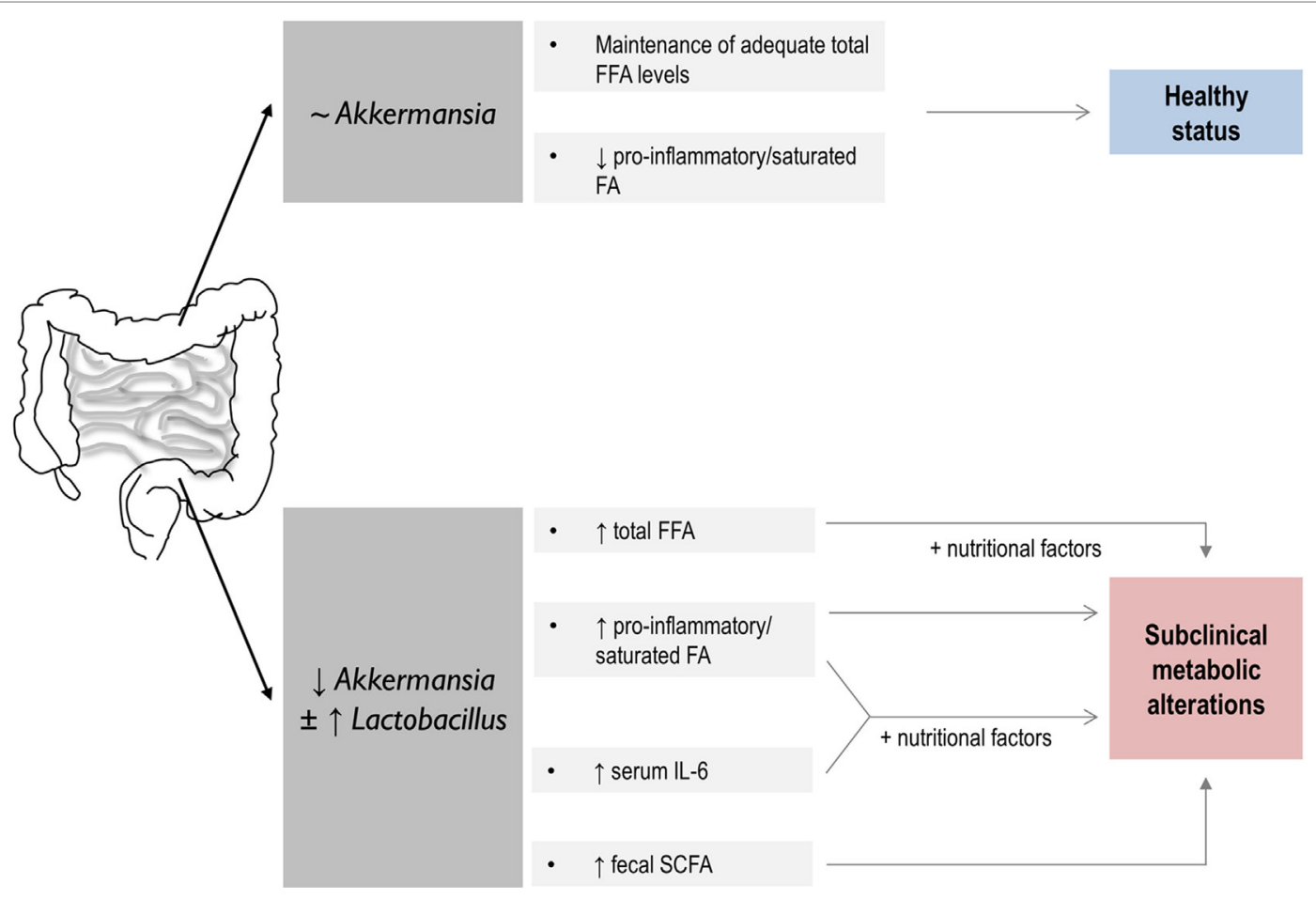

FIGURE 4 | Summary of the global findings of the present study and proposed hypothesis on their potential impact in human health. A link between host lipid metabolism and gut microbiota with potential consequences for the human health is disclosed. On the one hand, above a certain value, Akkermansia levels seem to play a role in the maintenance of the serum free fatty acids (FFA) pool in healthy individuals. Decreased Akkermansia abundance in the gut microbiota, which seems to coincide in our study with increased Lactobacillus counts, is associated with an altered serum FFA profile, hallmarked by striking quantitative and qualitative differences in FFA as well as in IL-6 serum levels and intestinal short-chain fatty acid (SCFA) production. These features, and their interaction with nutritional factors, were related to an increased prevalence of subclinical metabolic alterations. Thus, a link between dysbalanced microbial populations, host lipid FFA metabolism, and human health may be suggested, SCFA production playing an important role.

(56), thereby suggesting that trophic interactions may underlie, at least in part, these opposite trends of both microorganisms. Actually, a negative effect on Akkermansia levels in the gut upon administration of a probiotic mixture was observed when Lactobacillus, but not other probiotic bacteria, were added to the mixture (57). Conversely, Akkermansia has been shown to increase the production of the antimicrobial peptide RegIII $\gamma$ by colonic epithelial cells (35). This peptide specifically targets Gram-positive bacteria, thus potentially accounting for the opposite trends between Akkermansia and Lactobacillus groups. In sum, it is feasible that Akkermansia may modulate the gut environment and some intestinal microbial populations through several mechanisms (58).

It has been reported that Akkermansia and Lactobacillus exhibited diverging trends in twins discordant for metabolic syndrome (40). Our results herein presented are in line with these findings and suggest that the altered composition of the intestinal microbiota may be found in subclinical stages in healthy subjects. A recent study in mice revealed that whereas the colonization by Akkermansia muciniphila shifted the intestinal mucosa gene expression profile toward gene pathways involved in immune tolerance and metabolic homeostasis, colonization by Lactobacillus plantarum resulted in an overexpression of genes involved in the metabolism of fatty acids, lipoprotein lipase being one of the most up-regulated genes (59). An enhanced enzymatic activity of this enzyme may contribute to explain the striking increase in serum FFA found in association with increased Lactobacillus abundance in our study. Actually, overexpression of lipoprotein lipase gene has been related to fatty acid accumulation and insulin resistance $(60,61)$. On the other hand, the balanced immune responses promoted upon Akkermansia colonization may also account for the increased IL-6 levels found in our study in subjects with diminished abundance of Akkermansia. This is also in accordance with the positive effect on the induction of regulatory $\mathrm{T}$ cells reported in mice administered Akkermansia (62). It must be noted that IL- 6 can promote a number of pleiotropic functions other than triggering inflammation. However, since we have focused our analysis in subjects with no previous diagnosis of chronic or immune-mediated conditions, our approach allowed us to gain insight into the relationships between Akkermansia, IL-6 and low-grade inflammation in healthy states. Nevertheless, these associations cannot be directly translated into pathological frameworks. Therefore, a study of the gut microbiota compositionIL-6 axis in disease stages remains to be elucidated.

Modulation by Akkermansia of genes involved in lipid metabolism seems to be mediated, at least in part, by the production of SCFA (63). Imbalanced microbial populations can have an impact on SCFA production, which can elicit different 
responses in the host metabolism and immune system (64). Interestingly, several authors have found increased SCFA in obesity and metabolic syndrome [reviewed in Ref. (65)], acetate and propionate being especially relevant in such alterations $(66,67)$. The associations found in our study between acetate and IL- 6 and that of the total SCFA with FFA serum levels strongly support the cross talk between gut microbiota, host metabolism and immune networks, highlighting a role for SCFA as important elements of this interplay.

The present study has a number of limitations that can be remarked. On the one hand, we have performed a targeted analysis of the gut microbial composition instead of a global profiling by $16 \mathrm{~S}$ rRNA gene sequences analysis. As a consequence, whether a reduced diversity underlies the present findings cannot be concluded. Similarly, a number of selected FFA and the major SCFA species were chosen for the analysis, based on their outmost relevance in several biological processes. In addition, the same concerns apply to the analysis of inflammatory mediators. Finally, a more precise characterization of dietary intakes may be needed to precisely account for the exact contribution of short-term nutrition to the FFA levels.

In conclusion, we reported an association between some intestinal microbial populations and the characteristics of the FFA profile in healthy middle-aged subject. Akkermansia and Lactobacillus groups seem to be connected to the health metabolic status of the host, the interplay with nutritional parameters playing a potential role (Figure 4). Finally, our findings provide some evidence on the role of SCFA as mediators of the cross talk in the gut microbiota-host lipid metabolism axis. Although our results point to a very early role of an altered microbial composition in the further development of metabolic disorders, prospective and long-term studies are needed to accurately address this possibility.

\section{AUTHOR CONTRIBUTIONS}

All the authors listed made substantial contributions to the design of the work, analysis, or interpretation of the results obtained;

\section{REFERENCES}

1. Håversen L, Danielsson KN, Fogelstrand L, Wiklund O. Induction of proinflammatory cytokines by long-chain saturated fatty acids in human macrophages. Atherosclerosis (2009) 202:382-93. doi:10.1016/j.atherosclerosis.2008. 05.033

2. Schaeffler A, Gross P, Buettner R, Bollheimer C, Buechler C, Neumeier M, et al. Fatty acid-induced induction of Toll-like receptor-4/nuclear factorkappaB pathway in adipocytes links nutritional signalling with innate immunity. Immunology (2009) 126:233-45. doi:10.1111/j.1365-2567.2008. 02892.x

3. Frommer KW, Schäffler A, Rehart S, Lehr A, Müller-Ladner U, Neumann E. Free fatty acids: potential proinflammatory mediators in rheumatic diseases. Ann Rheum Dis (2015) 74:303-10. doi:10.1136/annrheumdis-2013-203755

4. Honda KL, Lamon-Fava S, Matthan NR, Wu D, Lichtenstein AH. EPA and DHA exposure alters the inflammatory response but not the surface expression of Toll-like receptor 4 in macrophages. Lipids (2015) 50:121-9. doi:10.1007/s11745-014-3971-y

5. Hung AM, Booker C, Ellis CD, Siew ED, Graves AJ, Shintani A, et al. Omega-3 fatty acids inhibit the up-regulation of endothelial chemokines in maintenance hemodialysis patients. Nephrol Dial Transplant (2015) 30:266-74. doi:10.1093/ndt/gfu283 participated in the study design and data interpretation, reviewed the manuscript, and approved the final version; and agreed to be accountable for all aspects of the work in ensuring that questions related to the accuracy or integrity of any part of the work are appropriately investigated and resolved. JR-C and NS performed most of the experimental procedures. SG was involved in the nutritional assessments, anthropometrical measurements, and collection of samples. AM, MG, CR-G, and AS provided biological samples and financial support. JR-C and AS drafted the manuscript.

\section{ACKNOWLEDGMENTS}

The authors acknowledge the excellent technical assistance of Ana M. Hernández-Barranco (IPLA) with the analysis of SCFA in fecal samples as well as of the staff of the scientific core facilities from the University of Oviedo (Unidad de Espectrometría y Espectrofotometría, Servicios Científico-Técnicos, Universidad de Oviedo). The authors also show their deepest gratitude to all the study volunteers. The authors acknowledge support of the publication fee by the CSIC Open Access Publication Support Initiative through its Unit of Information Resources for Research (URICI).

\section{FUNDING}

This work was funded through the Grant GRUPIN14-043 "Microbiota Humana, Alimentación y Salud" funded by "Plan Regional de Investigación del Principado de Asturias," Asturias, Spain and by the grants AGL2010-14952 from Spanish "Plan Nacional I+D+I" and by Biopolis SL within the framework of the e-CENIT Project SENIFOOD from the Spanish Ministry of Science and Innovation. JR-C and NS benefit from postdoctoral contracts supported by the Grant GRUPIN14-043 and by a Clarín regional contract cofinanced by the Marie Curie CoFund European Program, respectively. Regional grants received cofounding from European Union FEDER funds.

6. Miles EA, Thies F, Wallace FA, Powell JR, Hurst TL, Newsholme EA, et al. Influence of age and dietary fish oil on plasma soluble adhesion molecule concentrations. Clin Sci (Lond) (2001) 100:91-100. doi:10.1042/cs1000091

7. Livingstone KM, Givens DI, Jackson KG, Lovegrove JA. Comparative effect of dairy fatty acids on cell adhesion molecules, nitric oxide and relative gene expression in healthy and diabetic human aortic endothelial cells. Atherosclerosis (2014) 234:65-72. doi:10.1016/j.atherosclerosis.2014.02.015

8. Serhan CN, Chiang N, Van Dyke TE. Resolving inflammation: dual antiinflammatory and pro-resolution lipid mediators. Nat Rev Immunol (2008) 8:349-61. doi: $10.1038 /$ nri2294

9. Boden G. Obesity and free fatty acids. Endocrinol Metab Clin North Am (2008) 37:635-46, viii-ix. doi:10.1016/j.ecl.2008.06.007

10. Cao H, Gerhold K, Mayers JR, Wiest MM, Watkins SM, Hotamisligil GS. Identification of a lipokine, a lipid hormone linking adipose tissue to systemic metabolism. Cell (2008) 134:933-44. doi:10.1016/j.cell.2008.07.048

11. Perreault M, Roke K, Badawi A, Nielsen DE, Abdelmagid SA, El-Sohemy A, et al. Plasma levels of 14:0, 16:0, 16:1n-7, and 20:3n-6 are positively associated, but 18:0 and 18:2n-6 are inversely associated with markers of inflammation in young healthy adults. Lipids (2014) 49:255-63. doi:10.1007/ s11745-013-3874-3

12. Aghdassi E, Ma DWL, Morrison S, Hillyer LM, Clarke S, Gladman DD, et al. Alterations in circulating fatty acid composition in patients with systemic 
lupus erythematosus: a pilot study. JPEN J Parenter Enteral Nutr (2011) 35:198-208. doi:10.1177/0148607110386378

13. Nishi SK, Kendall CWC, Bazinet RP, Bashyam B, Ireland CA, Augustin LSA, et al. Nut consumption, serum fatty acid profile and estimated coronary heart disease risk in type 2 diabetes. Nutr Metab Cardiovasc Dis (2014) 24:845-52. doi:10.1016/j.numecd.2014.04.001

14. Van Hees NJM, Giltay EJ, Geleijnse JM, Janssen N, Van Der Does W. DHA serum levels were significantly higher in celiac disease patients compared to healthy controls and were unrelated to depression. PLoS One (2014) 9:e97778. doi:10.1371/journal.pone.0097778

15. Dai L, Gonalves CMV, Lin Z, Huang J, Lu H, Yi L, et al. Exploring metabolic syndrome serum free fatty acid profiles based on GC-SIM-MS combined with random forests and canonical correlation analysis. Talanta (2015) 135:108-14. doi:10.1016/j.talanta.2014.12.039

16. Wang D-C, Sun C-H, Liu L-Y, Sun X-H, Jin X-W, Song W-L, et al. Serum fatty acid profiles using GC-MS and multivariate statistical analysis: potential biomarkers of Alzheimer's disease. Neurobiol Aging (2012) 33:1057-66. doi:10.1016/j.neurobiolaging.2010.09.013

17. Rodriguez-Carrio J, Alperi-Lopez M, López P, Ballina-Garcia FJ, Suárez A. Non-esterified fatty acids profiling in rheumatoid arthritis: associations with clinical features and Th1 response. PLoS One (2016) 11:e0159573. doi:10.1371/ journal.pone. 0159573

18. Tremaroli V, Bäckhed F. Functional interactions between the gut microbiota and host metabolism. Nature (2012) 489:242-9. doi:10.1038/ nature 11552

19. Scott KP, Gratz SW, Sheridan PO, Flint HJ, Duncan SH. The influence of diet on the gut microbiota. Pharmacol Res (2013) 69:52-60. doi:10.1016/j. phrs.2012.10.020

20. Cani PD, Bibiloni R, Knauf C, Waget A, Neyrinck AM, Delzenne NM, et al. Changes in gut microbiota control metabolic endotoxemia-induced inflammation in high-fat diet-induced obesity and diabetes in mice. Diabetes (2008) 57:1470-81. doi:10.2337/db07-1403

21. Bäckhed F, Ding H, Wang T, Hooper LV, Koh GY, Nagy A, et al. The gut microbiota as an environmental factor that regulates fat storage. Proc Natl Acad Sci U S A (2004) 101:15718-23. doi:10.1073/pnas.0407076101

22. Samuel BS, Shaito A, Motoike T, Rey FE, Backhed F, Manchester JK, et al. Effects of the gut microbiota on host adiposity are modulated by the shortchain fatty-acid binding G protein-coupled receptor, Gpr41. Proc Natl Acad Sci U S A (2008) 105:16767-72. doi:10.1073/pnas.0808567105

23. Sharon G, Garg N, Debelius J, Knight R, Dorrestein PC, Mazmanian SK. Specialized metabolites from the microbiome in health and disease. Cell Metab (2014) 20:719-30. doi:10.1016/j.cmet.2014.10.016

24. Pizarro C, Arenzana-Rámila I, Pérez-Del-Notario N, Pérez-Matute P, González-Sáiz JM. Plasma lipidomic profiling method based on ultrasound extraction and liquid chromatography mass spectrometry. Anal Chem (2013) 85:12085-92. doi:10.1021/ac403181c

25. Cuervo A, Hevia A, López P, Suárez A, Sánchez B, Margolles A, et al. Association of polyphenols from oranges and apples with specific intestinal microorganisms in systemic lupus erythematosus patients. Nutrients (2015) 7:1301-17. doi:10.3390/nu7021301

26. Centro de Enseñanza Superior de Nutrición Humana y Dietética (CESNID). Tablas de composición de alimentos por medidas caseras de consumo habitual en España. Barcelona: McGraw-Hill Publicaciones y Ediciones la Univ Barcelona (2008).

27. Hevia A, Milani C, López P, Cuervo A, Arboleya S, Duranti S, et al. Intestinal dysbiosis associated with systemic lupus erythematosus. mBio (2014) 5: e01548-14. doi:10.1128/mBio.01548-14

28. Salazar N, López P, Valdés L, Margolles A, Suárez A, Patterson AM, et al. Microbial targets for the development of functional foods accordingly with nutritional and immune parameters altered in the elderly. J Am Coll Nutr (2013) 32:399-406. doi:10.1080/07315724.2013.827047

29. Valdés L, Gullón P, Salazar N, Rios-Covián D, González-Muñoz MJ, Parajó JC, et al. Population dynamics of some relevant intestinal microbial groups in human fecal batch cultures with added fermentable xylooligosaccharides obtained from rice husks. BioResources (2013) 8:2429-41.

30. Arboleya S, Binetti A, Salazar N, Fernández N, Solís G, HernándezBarranco A, et al. Establishment and development of intestinal microbiota in preterm neonates. FEMS Microbiol Ecol (2012) 79:763-72. doi:10.1111/j.1574-6941.2011.01261.x
31. Fernández-Navarro T, Salazar N, Gutiérrez-Díaz I, de Los Reyes-Gavilán CG, Gueimonde M, González S. Different intestinal microbial profile in overweight and obese subjects consuming a diet with low content of fiber and antioxidants. Nutrients (2017) 9:E551. doi:10.3390/nu9060551

32. YatsunenkoT,ReyFE,ManaryMJ,TrehanI,Dominguez-BelloMG,ContrerasM, et al. Human gut microbiome viewed across age and geography. Nature (2012) 486:222-7. doi:10.1038/nature11053

33. Eckburg PB, Bik EM, Bernstein CN, Purdom E, Dethlefsen L, Sargent M, et al. Diversity of the human intestinal microbial flora. Science (2005) 308:1635-8. doi:10.1126/science.1110591

34. Derrien M, Belzer C, de Vos WM. Akkermansia muciniphila and its role in regulating host functions. Microb Pathog (2016) 106:171-81. doi:10.1016/j. micpath.2016.02.005

35. Everard A, Belzer C, Geurts L, Ouwerkerk JP, Druart C, Bindels LB, et al. Cross-talk between Akkermansia muciniphila and intestinal epithelium controls diet-induced obesity. Proc Natl Acad Sci U S A (2013) 110:9066-71. doi:10.1073/pnas.1219451110

36. Schneeberger M, Everard A, Gómez-Valadés AG, Matamoros S, Ramírez S, Delzenne NM, et al. Akkermansia muciniphila inversely correlates with the onset of inflammation, altered adipose tissue metabolism and metabolic disorders during obesity in mice. Sci Rep (2015) 5:16643. doi:10.1038/ srep16643

37. Caesar R, Tremaroli V, Kovatcheva-Datchary P, Cani PD, Bäckhed F. Crosstalk between gut microbiota and dietary lipids aggravates WAT inflammation through TLR signaling. Cell Metab (2015) 22:658-68. doi:10.1016/j. cmet.2015.07.026

38. Dao MC, Everard A, Aron-Wisnewsky J, Sokolovska N, Prifti E, Verger EO, et al. Akkermansia muciniphila and improved metabolic health during a dietary intervention in obesity: relationship with gut microbiome richness and ecology. Gut (2016) 65:426-36. doi:10.1136/gutjnl-2014-308778

39. Cani PD, Amar J, Iglesias MA, Poggi M, Knauf C, Bastelica D, et al. Metabolic endotoxemia initiates obesity and insulin resistance. Diabetes (2007) 56:1761-72. doi:10.2337/db06-1491

40. Lim MY, You HJ, Yoon HS, Kwon B, Lee JY, Lee S, et al. The effect of heritability and host genetics on the gut microbiota and metabolic syndrome. Gut (2016) 66:1031-8. doi:10.1136/gutjnl-2015-311326

41. Png CW, Lindén SK, Gilshenan KS, Zoetendal EG, McSweeney CS, Sly LI, et al. Mucolytic bacteria with increased prevalence in IBD mucosa augment in vitro utilization of mucin by other bacteria. Am J Gastroenterol (2010) 105:2420-8. doi:10.1038/ajg.2010.281

42. Zhang X, Shen D, Fang Z, Jie Z, Qiu X, Zhang C, et al. Human gut microbiota changes reveal the progression of glucose intolerance. PLoS One (2013) 8:e71108. doi:10.1371/journal.pone.0071108

43. Leutner M, Göbl C, Wielandner A, Howorka E, Prünner M, Bozkurt L, et al. Clinical and metabolic characteristics of treated hyperlipidemic patients additionally affected by subclinical hyperglycemia. Lipids Health Dis (2016) 15:10. doi:10.1186/s12944-016-0180-0

44. Viscogliosi G, Andreozzi P, Marigliano V. Associations of plasma glucose levels and traits of metabolic syndrome with carotid intima media thickness in nondiabetic elderly subjects: are they mediated by insulin resistance? Metab Syndr Relat Disord (2013) 11:41-5. doi:10.1089/met.2012.0087

45. Fontbonne A, Eschwège E, Cambien F, Richard JL, Ducimetière P, Thibult N, et al. Hypertriglyceridaemia as a risk factor of coronary heart disease mortality in subjects with impaired glucose tolerance or diabetes. Results from the 11-year follow-up of the Paris Prospective Study. Diabetologia (1989) 32:300-4. doi:10.1007/BF00265546

46. Fontbonne A, Thibult N, Eschwège E, Ducimetière P. Body fat distribution and coronary heart disease mortality in subjects with impaired glucose tolerance or diabetes mellitus: the Paris Prospective Study, 15-year follow-up. Diabetologia (1992) 35:464-8. doi:10.1007/BF02342445

47. Levitan EB, Song Y, Ford ES, Liu S. Is nondiabetic hyperglycemia a risk factor for cardiovascular disease? Arch Intern Med (2004) 164:2147. doi:10.1001/ archinte.164.19.2147

48. Pistrosch F, Natali A, Hanefeld M. Is hyperglycemia a cardiovascular risk factor? Diabetes Care (2011) 34:S128-31. doi:10.2337/dc11-s207

49. Armougom F, Henry M, Vialettes B, Raccah D, Raoult D. Monitoring bacterial community of human gut microbiota reveals an increase in Lactobacillus in obese patients and methanogens in anorexic patients. PLoS One (2009) 4:e7125. doi:10.1371/journal.pone.0007125 
50. Million M, Angelakis E, Maraninchi M, Henry M, Giorgi R, Valero R, et al. Correlation between body mass index and gut concentrations of Lactobacillus reuteri, Bifidobacterium animalis, Methanobrevibacter smithii and Escherichia coli. Int J Obes (Lond) (2013) 37:1460-6. doi:10.1038/ijo.2013.20

51. Million M, Maraninchi M, Henry M, Armougom F, Richet H, Carrieri P, et al. Obesity-associated gut microbiota is enriched in Lactobacillus reuteri and depleted in Bifidobacterium animalis and Methanobrevibacter smithii. Int J Obes (Lond) (2012) 36:817-25. doi:10.1038/ijo.2011.153

52. Million M, Thuny F, Angelakis E, Casalta J-P, Giorgi R, Habib G, et al. Lactobacillus reuteri and Escherichia coli in the human gut microbiota may predict weight gain associated with vancomycin treatment. Nutr Diabetes (2013) 3:e87. doi:10.1038/nutd.2013.28

53. Zeng H, Liu J, Jackson MI, Zhao F-Q, Yan L, Combs GF. Fatty liver accompanies an increase in Lactobacillus species in the hind gut of C57BL/6 mice fed a high-fat diet. J Nutr (2013) 143:627-31. doi:10.3945/jn.112.172460

54. Consolandi C, Turroni S, Emmi G, Severgnini M, Fiori J, Peano C, et al. Behçet's syndrome patients exhibit specific microbiome signature. Autoimmun Rev (2015) 14:269-76. doi:10.1016/j.autrev.2014.11.009

55. Wang J, Tang H, Zhang C, Zhao Y, Derrien M, Rocher E, et al. Modulation of gut microbiota during probiotic-mediated attenuation of metabolic syndrome in high fat diet-fed mice. ISME J (2015) 9:1-15. doi:10.1038/ismej.2014.99

56. Tran THT, Boudry C, Everaert N, Théwis A, Portetelle D, Daube G, et al. Adding mucins to an in vitro batch fermentation model of the large intestine induces changes in microbial population isolated from porcine feces depending on the substrate. FEMS Microbiol Ecol (2016) 92:fiv165. doi:10.1093/ femsec/fiv165

57. Alard J, LehrterV, Rhimi M, Mangin I, Peucelle V, Abraham A-L, et al. Beneficial metabolic effects of selected probiotics on diet-induced obesity and insulin resistance in mice are associated with improvement of dysbiotic gut microbiota. Environ Microbiol (2016) 18:1484-97. doi:10.1111/1462-2920.13181

58. Belzer C, de Vos WM. Microbes inside-from diversity to function: the case of Akkermansia. ISME J (2012) 6:1449-58. doi:10.1038/ismej.2012.6

59. Derrien M, Van Baarlen P, Hooiveld G, Norin E, Müller M, de Vos WM. Modulation of mucosal immune response, tolerance, and proliferation in mice colonized by the mucin-degrader Akkermansia muciniphila. Front Microbiol (2011) 2:166. doi:10.3389/fmicb.2011.00166

60. Ferreira LD, Pulawa LK, Jensen DR, Eckel RH. Overexpressing human lipoprotein lipase in mouse skeletal muscle is associated with insulin resistance. Diabetes (2001) 50:1064-8. doi:10.2337/diabetes.50.5.1064
61. Kim JK, Fillmore JJ, Chen Y, Yu C, Moore IK, Pypaert M, et al. Tissue-specific overexpression of lipoprotein lipase causes tissue-specific insulin resistance. Proc Natl Acad Sci U S A (2001) 98:7522-7. doi:10.1073/pnas.121164498

62. Shin N-R, Lee J-C, Lee H-Y, Kim M-S, Whon TW, Lee M-S, et al. An increase in the Akkermansia spp. population induced by metformin treatment improves glucose homeostasis in diet-induced obese mice. Gut (2014) 63:727-35. doi:10.1136/gutjnl-2012-303839

63. Lukovac S, Belzer C, Pellis L, Keijser BJ, de Vos WM, Montijn RC, et al. Differential modulation by Akkermansia muciniphila and Faecalibacterium prausnitzii of host peripheral lipid metabolism and histone acetylation in mouse gut organoids. mBio (2014) 5(4):e01438-14. doi:10.1128/mBio.01438-14

64. Brestoff JR, Artis D. Commensal bacteria at the interface of host metabolism and the immune system. Nat Immunol (2013) 14:676-84. doi:10.1038/ ni. 2640

65. Ríos-Covián D, Ruas-Madiedo P, Margolles A, Gueimonde M, de Los Reyes-Gavilán CG, Salazar N. Intestinal short chain fatty acids and their link with diet and human health. Front Microbiol (2016) 7:185. doi:10.3389/ fmicb.2016.00185

66. Salazar N, Dewulf EM, Neyrinck AM, Bindels LB, Cani PD, Mahillon J, et al. Inulin-type fructans modulate intestinal Bifidobacterium species populations and decrease fecal short-chain fatty acids in obese women. Clin Nutr (2015) 34:501-7. doi:10.1016/j.clnu.2014.06.001

67. Vrieze A, Holleman F, Zoetendal EG, de Vos WM, Hoekstra JBL, Nieuwdorp M. The environment within: how gut microbiota may influence metabolism and body composition. Diabetologia (2010) 53:606-13. doi:10.1007/ s00125-010-1662-7

Conflict of Interest Statement: The authors declared no potential competing financial interests concerning this study. Funders had no role in study conception, design, analysis of the results, or decision to publish.

Copyright (C) 2017 Rodríguez-Carrio, Salazar, Margolles, González, Gueimonde, de los Reyes-Gavilán and Suárez. This is an open-access article distributed under the terms of the Creative Commons Attribution License (CC BY). The use, distribution or reproduction in other forums is permitted, provided the original author(s) or licensor are credited and that the original publication in this journal is cited, in accordance with accepted academic practice. No use, distribution or reproduction is permitted which does not comply with these terms. 